\title{
Device Process and Circuit Application Interaction for Harsh Electronics: Hf-In-Zn-O Thin Film Transistors as an Example
}

Chih-Hsiang $\mathrm{Ho}^{1 *}$, Dung-Sheng Tsai ${ }^{2}$, Chao Lu ${ }^{3}$, Soo Youn Kim ${ }^{4}$, Selin Mungan ${ }^{1}$, Shih-Guo Yang ${ }^{2}$, Yuanzhi Zhang ${ }^{3}$ and Jr$\mathrm{Hau} \mathrm{He}^{5 *}$

\begin{abstract}
The effects of Hf content on the radiation hardness of Hf-In-Zn-O thin-film transistors (HIZO TFTs) and HIZO TFTbased circuits are systemically examined. The evaluated circuits, including current-starved ring oscillator, energy harvesting and RF circuits are essential for space electronic systems. It is shown that HIZO TFTs with low Hf concentration have better initial performance while TFTs with high Hf concentration are more stable against radiation. On the other hand, for circuit application, the stable HIZO TFTs are not necessarily preferred for all circuits. The work demonstrates that understanding the device-circuit interactions is necessary for device optimization and circuit reliability improvements for harsh electronic systems.
\end{abstract}

Index Terms - Hf-In-Zn-O (HIZO), thin film transistor (TFT), harsh electronics.

\section{Introduction}

Due to the increasing demand for circuits to operate in extreme environment from the industries, such as oil, gas, aerospace, military companies, harsh electronics have drawn more and more attention [1-7]. While much insight on device physics of harsh electronics has been gained in recent years, understanding of the relation between device process and circuit performance has been rather limited [8-9]. In particular, it is expected that the requirements for device characteristics for better circuit reliability may vary depending on the circuit structure.

Therefore, in this work, using HIZO TFTs (which show better stability and performance as compared to other oxideTFT counterparts) in space electronic system as an example, we systemically examined the effects of Hf content on the radiation hardness of HIZO TFTs [10-12]. Further, the dependence of circuit performance on the Hf content of HIZO TFT are also explored. The evaluated HIZO TFT-based circuits, including current-starved ring oscillator, energy harvesting and RF circuits are essential for space electronic systems [13-14]. Our results indicate that HIZO TFTs with low Hf concentration feature a better initial performance while HIZO TFTs with higher Hf concentration shows high stability under proton exposure. In circuit level, the device stability is proven to be critical for oscillator application. On the contrary, for RF and power convertor circuits, the weak radiation resistance of low Hf concentration HIZO TFTs is preferred.

C.-H. Ho and S. Mungan are with the Purdue University, West Lafayette, IN, 47907-6130, USA (e-mail: hjscow@ gmail.com).

D.-S. Tsai and S.-G. Yang are with 2Institute of Photonics and Optoelectronics, National Taiwan University, Taipei 10617, Taiwan (e-mail: dungsheng@gmail.com).

C. Lu and Y Zhang are with the Department of ECE, Southern Illinois University, Carbondale, IL, 62901, USA (e-mail: eeluchao@gmail.com).

S. Y. Kim is with Department of Semiconductor Science, Dongguk University, Seoul 04620, South Korea (e-mail: sookim.kr@gmail.com).

J.-H. He is with Computer, Electrical, and Mathematical Sciences and Engineering (CEMSE) Division, King Abdullah University of Science \& Technology (KAUST), Thuwal 23955-6900, Saudi Arabia (e-mail: jrhau.he@kaust.edu.sa).

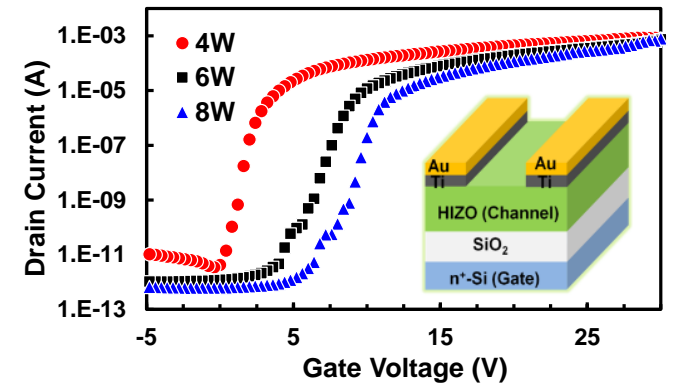

Fig. 1 The transfer characteristics of HIZO TFTs with DX Hf target sputtering power from $4 \mathrm{~W}$ to $8 \mathrm{~W}$. The inset is the schematic diagram of HIZO TFT.

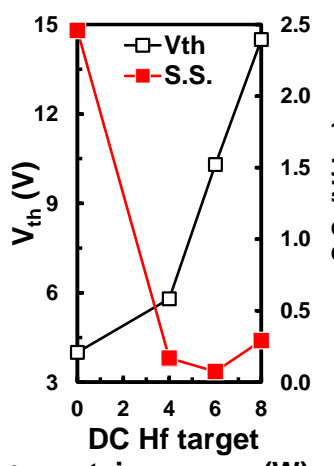

(a) sputeing power (W)

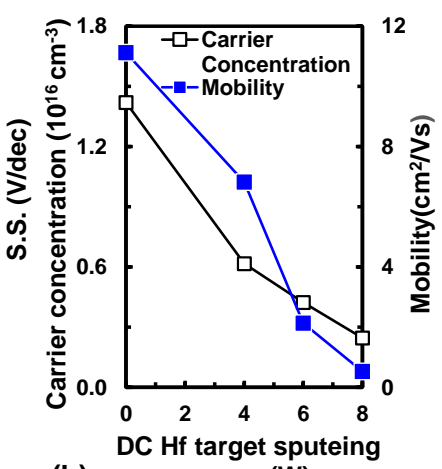

(b) power (W)
Fig. 2 (a) Threshold voltage $\left(V_{t h}\right)$, Subthreshold slope (S.S.), (b) carrier concentration and Mobility as a function of DC Hf target sputtering power.

\section{Fabrication \& Device Characteristics}

Fig. 1 shows the schematic of the HIZO TFTs. Thermally grown 90 -nm-thick $\mathrm{SiO} 2$ on $\mathrm{n}+-\mathrm{Si}(\sim 0.01 \Omega \mathrm{cm})$ substrates is adopted for a gate dielectric/electrode. Prior to the deposition of HIZO thin films, the substrate was cleaned with acetone, IPA and de-ionized water. A 35-nm-thick HIZO thin film was grown by radio-frequency. To investigate the influence of $\mathrm{Hf}$ concentration on the radiation hardness of HIZO TFTs, a range of DC Hf target sputtering power $(4 \mathrm{~W}-8 \mathrm{~W})$ was applied in the fabrication process. After the deposition of channel material, the patterning of source and drain electrodes was defined using photolithography. Then, 20-nm-thick Ti and 80-nm-thick Au with $5 \mu \mathrm{m}$ wide and $200 \mu \mathrm{m}$ long were deposited for source/drain electrodes by e-gun, followed by photolithography/lifted off processes. The channel length $(L)$ is $5 \mu \mathrm{m}$, and channel width $(W)$ is $200 \mu \mathrm{m}$. Finally, the fabricated TFTs were annealed at $150{ }^{\circ} \mathrm{C}$ for 1 hour in N2 ambient. After the fabrication process of HIZO TFTs, the electrical characteristics of devices were measured by a Keithley 4200 semiconductor parameter analyzer at room temperature in the dark. Moreover, for radiation resistance testing, HIZO TFTs were irradiated with a $2-\mathrm{MeV}$ proton with the fluences ranging from $10^{13}$ to $10^{15} \mathrm{~cm}^{-2}$ from a $3-\mathrm{MV}$ 


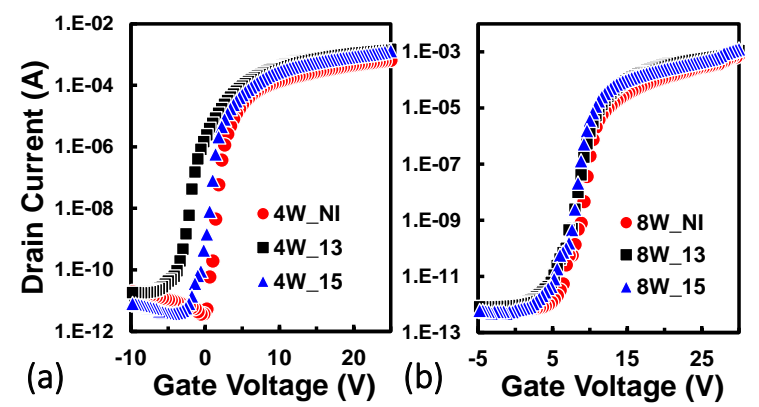

Fig. $3 V_{G S}-I_{D S}$ curves of HIZO TFTs under (a) $4 \mathrm{~W}$ and (b) $8 \mathrm{~W}$ DC Hf target sputtering power with three irradiation fluence conditions (i.e. no irradiation, $10^{13} \mathrm{~cm}^{-2}$ and $10^{15} \mathrm{~cm}^{-2}$ proton irradiation fluence). The $V_{G S}-I_{D S}$ is measured in the dark box at room temperature.

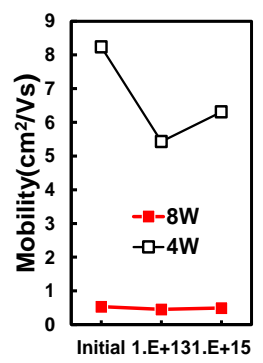

(a) Fluences $\left(\mathrm{cm}^{-2}\right)$

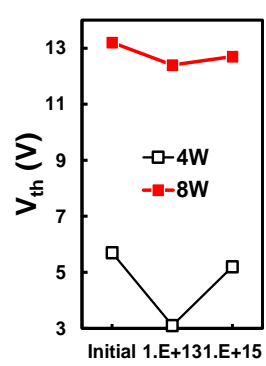

(b) Fluences $\left(\mathrm{cm}^{-2}\right)$

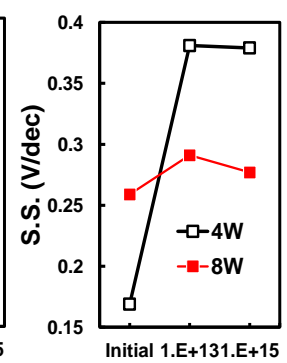

(c) Fluences $\left(\mathrm{cm}^{-2}\right)$
Fig. 4 (a) $\mu_{F E}$, (b) $V_{t h}$ and (c) $S . S$ as a function of $2-\mathrm{MeV}$ proton irradiation fluence with two different DX Hf target sputtering power $(4 \mathrm{~W}$ and $8 \mathrm{~W})$. The results are extracted from the $I-V$ curves of HIZO TFTs in Fig. 3.

tandem accelerator (NEC 9SDH-2, National Electrostatics Corporation). Fig. 1 shows the transfer characteristics of HIZO TFTs with DC Hf target sputtering power from $4 \mathrm{~W}$ to $8 \mathrm{~W}$, while the inset of Fig.1 illustrates the schematic of the asfabricated HIZO TFTs. It is clearly shown in Fig. 2 that when DC Hf target sputtering power increases (i.e., the Hf concentration increases), the threshold voltage $\left(V_{t h}\right)$ shifts positively while subthreshold slope (S.S.), carrier concentration $\left(N_{c}\right)$, mobility $\left(\mu_{F E}\right)$ and on-current level decreases. The observed decrease in S.S. with the increase in Hf concentration is attributed to the reduction in interface and deep trap states. Furthermore, the Hf content-dependent carrier concentration indicates that Hf ions may act as charge-carrier suppressors due to its high oxygen binding energy. Moreover, the decreased $\mu_{F E}$ can be attributed to the heavy weight of Hf content that suppresses the carrier diffusion. These results demonstrate the strong dependence of HIZO TFT characteristics on the Hf concentration.

\section{Device-level Radiation Resistance Test}

For radiation resistance testing, HIZO TFTs were irradiated with a $2-\mathrm{MeV}$ proton beam with the fluences ranging from $10^{13}$ to $10^{15} \mathrm{~cm}^{-2}$ from a $3-\mathrm{MV}$ tandem accelerator. The $V_{G S}-I_{D S}$ curves of HIZO TFTs with $4 \mathrm{~W}$ and $8 \mathrm{~W}$ DC target sputtering power under different proton fluences are shown in Fig. 3. The results demonstrate narrow distribution for the case of $8 \mathrm{~W}$. The device characteristics of HIZO including, $\mu_{F E}, V_{t h}$ and S.S. are extracted and shown in Fig.4. It is clear in Fig. 4(a) that after

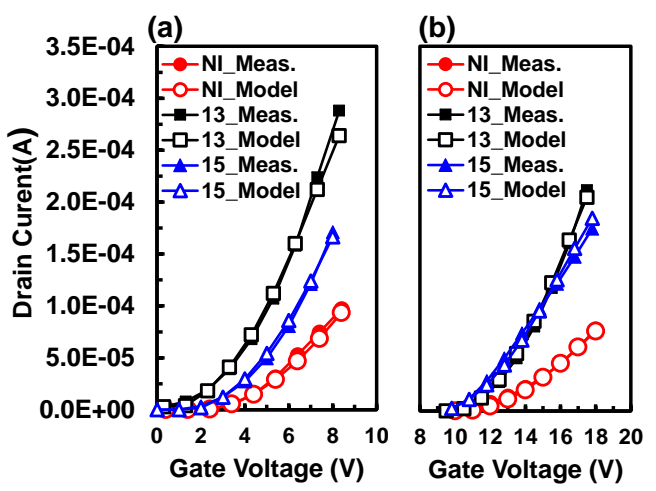

Fig. 5 Comparison of $I-V$ curves from Level 62 RPI SPICE model and experimental results for HIZO TFTs under (a) 4W and (b) $8 \mathrm{~W}$ DC Hf target sputtering power with three irradiation fluence conditions (i.e. no irradiation, $10^{13} \mathrm{~cm}^{-2}$ and $10^{15} \mathrm{~cm}^{-2}$ proton irradiation fluence).

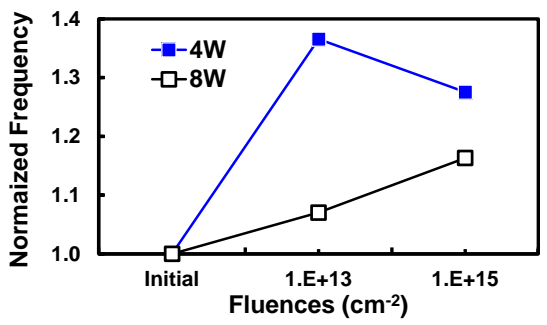

Fig. 6 The output frequency of a three-stage current-starved ring oscillator [13] as a function of proton irradiation fluence for $4 \mathrm{~W}$ and $8 \mathrm{~W}$ Hf target sputtering power conditions.

proton exposure at a fluence of $10^{13} \mathrm{~cm}^{-2}$, a reduction in $\mu_{F E}$ was observed. The reduction is due to the increase in lattice and Coulomb scattering causing from the rise of protoninduced trap density in the channel/gate oxide interface and/or in the channel region. Moreover, the increased trap density leads to the negative directional shift of $V_{t h}$ and the increased $S . S$ as shown in Fig. 4(b) and 4(c). It is worth noting that, as the proton irradiation fluence increases to $10^{15} \mathrm{~cm}^{-2}$, the drain current of HIZO TFTs is recovered. These results suggest that recrystallization occurs due to the high substrate temperature during the ultra-high fluence proton radiation process (i.e., self-annealing effects). On the other hand, as shown in Fig. 3(b), the transfer characteristics of 8W TFTs are insensitive to the proton irradiation fluences. Clearly, the high $\mathrm{Hf}$ concentration makes the device more stable under proton irradiation. It can be attributed to the fact that the strong chemical bond in HIZO compounds due to the high oxygen binding energy of Hf, leads to the superior stability of HIZO TFTs under proton irradiation.

\section{Device Process and Circuit Application Interaction}

To study the effect of radiation on HIZO TFT-based circuits, HSPICE models were extracted and verified with measured device $V_{G S}-I_{D S}$ data for circuit simulation. As shown in Fig. 5, the $V_{G S}-I_{D S}$ results from Level 62 RPI SPICE model match well with measurement data.

Having the HSPICE model, we are able to examine and compare the radiation hardness of the HIZO TFT-based 
(a)

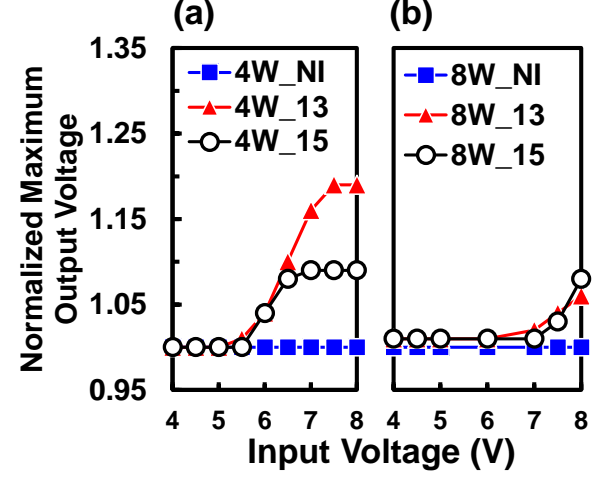

(c)

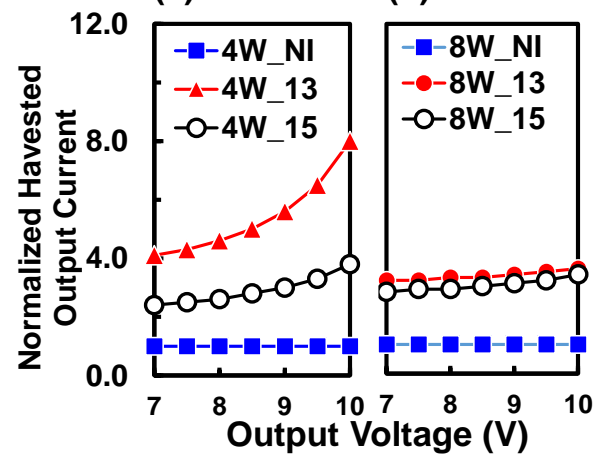

Fig. 7 Normalized maximum output voltage of a power converter circuit [14] as a function of input voltage with HIZO TFTs under (a) $4 \mathrm{~W}$ and (b) $8 \mathrm{~W}$ DC Hf target sputtering power and three irradiation fluence conditions. Normalized harvested output current of a power converter circuit as a function of output voltage with HIZO TFTs under (c) $4 \mathrm{~W}$ and (d) $8 \mathrm{~W}$ DC Hf target sputtering power and three irradiation fluence conditions.
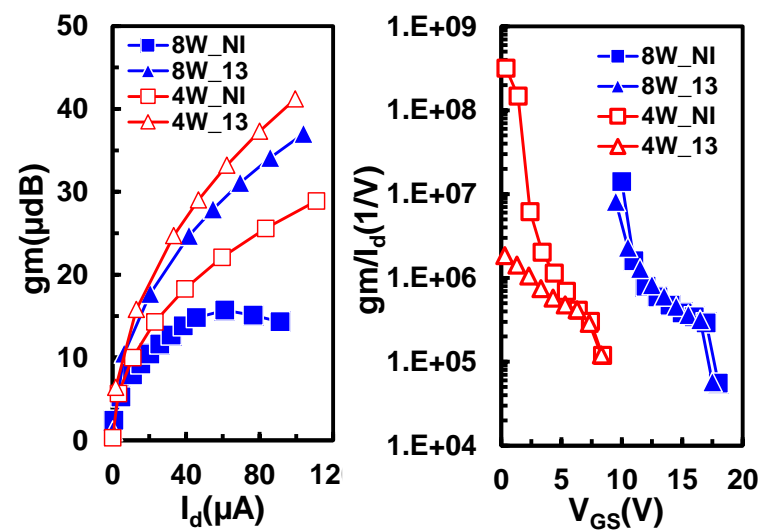

Fig. 8 Comparison of (a) cutoff frequency $\left(f_{T}\right)$ (b) normalized trans-conductance $\left(g_{m} / I_{D S}\right)$ for HIZO TFT-based RF circuit under $4 \mathrm{~W}$ and $8 \mathrm{~W}$ DC Hf target sputtering power with two irradiation fluence conditions (i.e. no irradiation and $10^{13} \mathrm{~cm}^{-2}$ proton irradiation fluence).

circuits including current-starved ring oscillator, energy harvesting and RF circuits [13-14]. Fig. 6 plots one of the most important characteristics of a three-stage current-starved ring oscillator, the oscillation frequency. Ring oscillators with $4 \mathrm{~W}$ TFTs exhibit a larger variation (37\%) on oscillation frequency than $8 \mathrm{~W}$ TFTs $(16 \%)$ after irradiation which is attributed to the larger $V_{t h}$ variation of $4 \mathrm{~W}$ TFTs. This observation suggests that $8 \mathrm{~W}$ TFT is preferred in this application since a stable output frequency is essential for oscillator circuits.
In a charge pump circuit, the most important characteristics, voltage boosting capability (i.e., maximum output voltage) and power transfer capability (i.e., harvested output current) are mainly determined by on-resistance of a transistor. As shown in Fig. 7, the maximum output voltage and harvested output current of charge pump increase after irradiation for both cases $(4 \mathrm{~W} \& 8 \mathrm{~W})$. Thus, power converter circuit benefits from proton radiation since $V_{t h}$ (as well as on-resistance) becomes smaller after irradiation. Note that, similar to device results, the maximum output voltage and harvested output current of charge pump restore back towards no irradiation case when exposing to $10^{15} \mathrm{~cm}^{-2}$ irradiation due to self-annealing effects, as shown in Fig. 3. Overall, in contrast to oscillator circuit, energy harvesting systems favor 4W HIZO TFTs. These results stress the importance in understanding device-circuit interactions for developing harsh electronic systems.

Finally, to evaluate the impacts of radiation on RF performances of a HIZO TFT, cutoff frequency $\left(f_{T}\right)$ and device normalized trans-conductance $\left(g_{m} / I_{D S}\right)$ extracted from the measured $V_{G S}-I_{D S}$ data in Fig. 3. As shown in Fig. 8(a) and 8(b), although $f_{T}$ and $g m / I_{D S}$ of $4 \mathrm{~W}$ TFTs are higher than those of $8 \mathrm{~W}$ TFTs due to the higher mobility, the $4 \mathrm{~W}$ TFTs clearly show larger variation with different irradiation fluence conditions on $f_{T}$ and $g m / I_{D S}$ than $8 \mathrm{~W}$ TFTs. It should be noted that the variation of device metrics (such as $V_{t h}, f_{T}$ and $g m / I_{D S}$ ) in analog and RF circuits require increased guard-banding at the design phase, resulting in the higher power consumption.

\section{Conclusion}

For the first time, the interaction between HIZO TFT process and HIZO TFT-based circuits were examined for harsh applications. We validated that HIZO TFTs with low Hf concentration can achieve better device performance while HIZO TFTs with higher Hf concentration exhibit high stability under proton exposure. On the other hand, for circuits, the stability is shown to be crucial for oscillator application. The weak radiation resistance of low Hf concentration HIZO TFTs, however, is preferred for RF and power convertor circuits. The device-circuit interaction results in this work give insights not only on process optimization for harsh electronic devices, but also in developing high performance and robust harsh electronic circuits.

\section{References}

[1] D.-S. Tsai, W.-C. Lien, D.-H. Lien, K.-M. Chen, M.-L. Tsai, D G. Senesky, Y.-C. Yu, A. P. Pisano and J.-H. He, "Solar-Blind Photodetectors for Harsh Electronics," Scientific Report, 4, 2628, Sep. 2013, DOI: 10.1038/srep02628.

[2] T. H. Huang, D.-S. Tsai, P.-K. Yang, W.-Y. Chang, J.-F. Kang, M.-J. Chen and J.-H. He, "Eliminating Surface effects via Employing Nitrogen Doping to Significantly Improve the Stability and Reliability of ZnO Resistive Memory," Mater. Chem. C, Issue 45, pp. 7593-7597, 2013, DOI:10.1039/C3TC31542H.

[3] D.-S. Tsai, D.-H. Lien, M.-L. Tsai, S.-H. Su, J.-J. Ke, Y.-C. Yu, L.-J. Li and J.-H. He, "Trilayered $\mathrm{MoS}_{2}$ Metal-Semiconductor-Metal Photodetectors: Photogain and Radiation Resistance," IEEE J. Select. Topics Quantum Elect., vol. 20, no. 1, 3800206, Jan./Feb., 2014, DOI: 10.1109/JSTQE.2013.2268383.

[4] P. Francis, C. Michel, D. Flandre and J. P. Colinge, "Radiationhard design for SOI MOS inverters," in IEEE Transactions on Nuclear Science, vol. 41, no. 2, pp. 402-407, Apr 1994. doi: 
$10.1109 / 23.281534$

[5] R. Picos, N. P. Papadopoulos, C.-H. Lee, A. Lope-Grifol, M. Roca, E. Isern, W. S. Wong and E. Garcia-Moreno, "Low dose radiation effects on a-Si:H TFTs," 2015 10th Spanish Conference on Electron Devices (CDE), Madrid, 2015, pp. 1-4. doi: 10.1109/CDE.2015.7087501

[6] V. Kilchytska, J. Alvarado, N. Collaert, R. Rooyakers, O. Militaru, G. Berger and D. Flandre, "Total-dose effects caused by high-energy neutrons and $\gamma$-rays in Multiple-Gate FETs," 2009 European Conference on Radiation and Its Effects on Components and Systems, Bruges, 2009, pp. 7-13. doi: 10.1109/RADECS.2009.5994544

[7] P. Francis, D. Flandre and J. P. Colinge, "Theoretical considerations for SRAM total-dose hardening," in IEEE Transactions on Nuclear Science, vol. 42, no. 2, pp. 83-91, Apr 1995. doi: 10.1109/23.372136.

[8] P. K. Yang, W.-Y. Chang, P.-Y. Teng, S.-F. Jeng, S.-J. Lin, P.-W. Chiu and J.-H. He, "Fully Transparent Resistive Memory Employing Graphene Electrodes for Eliminating Undesired Surface Effects" Proc. IEEE, vol. 101, issue 7, pp. 1732-1739, May, 2013, DOI: 10.1109/JPROC.2013.2260112.

[9] W. H. Jeong, G.-H. Kim, H.-S. Shin, B.-D. Ahn, H.-J. Kim, M.-K. Ryu, K.-B. Park, J.-B. Seon and S.-Y. Lee, "Investigating Addition Effect of Hafnium in InZnO Thin Film Transistors Using a Solution Process," Appl. Phys. Lett., vol. 96, issue 9, 093503, 2010, doi: http://dx.doi.org/10.1063/1.3340943.

[10] C.-J. Kim, S. Kim, J.-H. Lee, J.-S. Park, S. Kim, J. Park, E. Lee, J. Lee, Y. Park, J. H. Kim, S. T. Shin and U-I. Chung, "Amorphous hafnium-indium-zinc oxide semiconductor thin film transistors", Appl. Phys. Lett., vol. 95, no. 25, pp. 2103-2105, Dec. 2009, doi: http://dx.doi.org/10.1063/1.3275801

[11] H.-S. Kim, K.-B Park, K. S. Son, J. S. Park, W.-J. Maeng, T. S. Kim, K.-H. Lee, E. S. Kim, J. Lee, J. Suh, J.-B. Seon, M. K. Ryu, S. Y. Lee, K. Lee and S. Im, "The influence of sputtering power and $\mathrm{O} 2 / \mathrm{ArO} 2 / \mathrm{Ar}$ flow ratio on the performance and stability of HfIn-Zn-O thin film transistors under illumination", Appl. Phys. Lett., vol. 97, no. 10, pp. 102103-1-102103-3, Sep. 2010, doi: http://dx.doi.org/10.1063/1.3488823

[12] W. J. Maeng, J. S. Park, H.-S. Kim, K.-H. Lee, K. B. Park, K. S. Son, T. S. Kim, E. S. Kim, Y. N. Ham, M. Ryu and S. Y. Lee, "Photo and thermal stability enhancement of amorphous Hf-In-Zn-O thinfilm transistors by the modulation of back channel composition", Appl. Phys. Lett., vol. 98, no. 7, pp. 073503, 2011, doi: http://dx.doi.org/10.1063/1.3555446

[13] R. Jacob Baker, "CMOS: Circuit Design, Layout, and Simulation," $3^{\text {rd }}$ ed. New York: Wiley-IEEE press, 2010, pp. 562-563.

[14] E. S. Mungan, C. Lu, C.-H. Ho and K. Roy, "Effects of Deposition Process on Poly-Si Microscale Energy Harvesting Systems: A Simulation Study", IEEE Transactions on Electron Devices, vol. 63, no. 4, pp. 1650-1657, 2016, DOI: 10.1109/TED.2016.2535275 